\title{
(อ) OPEN ACCESS \\ Severe illicit gamma-hydroxybutyric acid withdrawal in a pregnant woman: what to do?
}

\author{
JC van Mechelen ำ, ${ }^{1}$ BAG Dijkstra, ${ }^{2,3}$ ACM Vergouwen ${ }^{1}$
}

\begin{abstract}
'Department of Psychiatry and Medical Psychology, OLVG Location West, Amsterdam, North Holland, the Netherlands ${ }^{2}$ Nijmegen Institute for ScientistPractitioners in Addiction (NISPA), Radboud University, Nijmegen, Gelderland, the Netherlands

${ }^{3}$ Research and development, Novadic-Kentron Addiction Care, Vught, Noord-Brabant, the Netherlands
\end{abstract}

\section{Correspondence to}

Dr JC van Mechelen;

joost.c.vanmechelen@gmail.com

Accepted 15 November 2019

\section{SUMMARY}

This case report presents the case of a 29 weeks pregnant woman in her late twenties who was seen at the emergency department of a hospital with a seizure of unknown cause. By anamnesis and heteroanamnesis the use of illicit gamma-hydroxybutyric acid (GHB) was revealed. Examination showed dilated pupils, sweating, tremor, tachycardia and bradyphrenic thinking. Subsequently, the seizure was indicated as a severe illicit GHB withdrawal symptom. Thereafter, treatment had to be decided on in the absence of evidence-based and practiced-based guidelines and treatment options for this specific patient population. Initially diazepam was started, which was later on substituted by sodium oxybate. Despite the critical professional situation the patient gave birth to a healthy daughter after 37 weeks of pregnancy.

\section{BACKGROUND}

In the current scientific literature the distinction between endogenous gamma-hydroxybutyric acid (GHB), the drug sodium oxybate and illicitly manufactured GHB is somewhat vague. ${ }^{1}$ This report refers to $\mathrm{GHB}$ as a reputed neurotransmitter and gamma aminobutyric acid (GABA) metabolite, that is endogenously synthesised by neurons in the brain $^{2}$; to sodium oxybate as the identical compound of GHB that is manufactured and distributed by the pharmaceutical industry as Xyrem; ${ }^{1}$ and to illicit $\mathrm{GHB}$ as all illegally manufactured GHB, sodium oxybate and street drug products that are intended to be GHB and/or (partially) contain the compound of GHB. ${ }^{1}$

Since illicit GHB is supposedly used for its euphoric and aphrodisiac-like effects, as well as for its enhancing effects on social behaviour, it has become progressively popular as a party drug. ${ }^{13}$ Besides the well-known positive effects associated with illicit $\mathrm{GHB}$, the association between its abuse and frequent overdoses, coma and (severe) intoxications resulting into recurrent presentations at emergency departments, as well as hospital admissions, are also well documented. ${ }^{4-7}$ Following the popularity of illicit GHB a general trend of increase in abuse was seen after it first emerged, even though overall prevalence numbers remain relatively low. ${ }^{8}$ However, since comparable systematic data on user prevalence between different countries is lacking, because of different user populations and patterns, a comprehensive overview is being hindered. ${ }^{9}$ For example, in the Netherlands, Norway, and the UK, the estimated prevalence for current illicit $\mathrm{GHB}$ use in the general population ranges from $0.1 \%$ to $1.3 \%$, whereas rates limited to specific subpopulations, for example partygoers, are considerably higher. ${ }^{10-12}$ Nevertheless, the profile of a European intoxicated by illicit GHB is being described as: a young man (mean age 31 years) in need of medical care for changed behaviour and a reduced consciousness, primarily during the weekend. ${ }^{9}$

Illicit GHB withdrawal symptoms can vary from mild symptoms consisting of tremors, tachycardia, anxiety, insomnia, hypertension, nausea and vomiting to severe symptoms including hallucinations, delusions, rhabdomyolysis, seizures and delirium. ${ }^{3}$ The severity of these withdrawal symptoms mostly depends on the frequency of ingestion of the illicit GHB and severe withdrawal can occur within an interval of 1-8 hours of ongoing usage. ${ }^{3}$

Despite that illicit GHB abuse poses a sizeable toxic health effect in the emergency setting, there are no specific epidemiologic descriptors known regarding the abuse of this drug during pregnancy. Even though this information lacks, illicit $\mathrm{GHB}$ abuse during pregnancy certainly presents a huge clinical challenge, partly due to the absence of evidence-based and practiced-based guidelines and treatment options for the (severe) withdrawal symptoms within this specific patient population.

This case report presents the case of a pregnant woman in her late twenties with illicit GHB withdrawal symptoms who was seen at the emergency department of a hospital in the Netherlands.

\section{CASE PRESENTATION}

Initially the woman, known with no relevant medical history and no family history, was presented to the neurologist with a seizure of unknown cause. However, after having admitted the use of illicit GHB, temazepam and baclofen, the liaison psychiatrist was consulted. In addition, examination revealed dilated pupils, sweating, tremor, tachycardia and bradyphrenic thinking.

\section{INVESTIGATIONS}

Further somatic screening revealed a large laceration of the chin as a result of a fall due to the seizure, a left-sided mandibular fracture with no (intra) cerebral bleeding on the CT scan, and a urinary drug test positive for benzodiazepines. Screening for illicit GHB was not part of the standard drugtesting panel. This is often the case in emergency settings, because of the short half-life of illicit GHB. 
In addition, heteroanamnesis disclosed that she was pregnant for 29 weeks. Subsequently, a cardiotocography was made, which showed no foetal stress.

\section{DIFFERENTIAL DIAGNOSIS}

This case concerns a healthy young woman with no previous medical history and without a relevant family history. She was presented while being intoxicated with polypharmacy with clinical symptomatology of dilated pupils, sweating, tremor, tachycardia and bradyphrenic thinking. In addition, somatic screening revealed no other significant leads. Thus, the seizure was classified as a severe withdrawal symptom. Thereafter, illicit GHB was prioritised by anamnesis and heteroanamneses as the main drug responsible for her withdrawal symptoms. Furthermore, anamnesis revealed that according to herself she had used a few tablets of temazepam and baclofen to temper the occurring illicit GHB withdrawal symptoms.

\section{TREATMENT}

After presentation the large laceration of the chin was immediately stitched. Thereafter, she was motivated for voluntary admission at the in-hospital psychiatry department for further treatment by a multidisciplinary team. After admission, diazepam orally was started to treat the withdrawal symptoms and by titration a total dosage of $15 \mathrm{mg}$ was reached within the first 24 hours. Thereafter, all withdrawal symptoms seemed to be in complete remission and the tapering-off of the diazepam to a dosage of $5 \mathrm{mg} /$ day was reached in over a week. During the tapering-off of the medication she continuously had a resting heart rate varying between 80 and 90 beats/min and she had trouble sleeping. For the latter mirtazapine $15 \mathrm{mg}$ was started without satisfactory result. On reaching a dosage of $2.5 \mathrm{mg} /$ day of the diazepam in more or less one and a half weeks she became agitated, disorganised and suffered from visual hallucinations. Following this acute change in mental status she suddenly presented herself with erratic behaviour by diving 'head first' onto the ground and by trying to burn her own leg with a lighter. Sodium oxybate was started in a dosage of $1050 \mathrm{mg}$ to treat the change in her mental state, which was indicated as an illicit GHB withdrawal delirium; by titration a dosage of $1950 \mathrm{mg}$ per every 2 hours was reached. At the same time diazepam was completely stopped. Parallel to treatment by medication she was voluntarily limited in her freedom of movement by physical restrains to prevent further self-harm. If at any point in time it was possible to alternatively provide adequate one-on-one supervision during this period, either by medical staff or family, the physical restrains were removed. In addition, a second CT scan of the head and neck was made, which showed no additional trauma. After the start of the sodium oxybate she stabilised quickly and it was possible to completely remove the physical restrains after approximately 5 days. Thereafter, the complete tapering-off of the sodium oxybate was reached in less than a week in steps of $300 \mathrm{mg}$ at a time. After treatment with sodium oxybate was stopped no signs of relapse of withdrawal symptoms were seen. Mirtazapine was continued with sufficient effect, since she did not have any further sleeping difficulties. Following her recovery of a withdrawal delirium she was operated with success for her mandibular fracture, while remaining admitted in the in-hospital psychiatry department for the duration of her pregnancy.

\section{OUTCOME AND FOLLOW-UP}

After having been pregnant for 37 weeks she was transferred to the obstetrics department to initiate the start of her labour, because of pre-eclampsia. She gave birth to a healthy daughter. Thereafter, both mother and daughter were discharged from the hospital in good condition, under the close supervision of the Child Protection Service and an outpatient psychiatry unit specialised in the treatment of addiction. Further follow-up after discharge is not available.

\section{DISCUSSION}

This case shows the difficulty of adequately recognising and treating illicit GHB withdrawal symptoms in a pregnant woman, in the absence of evidence-based and practiced-based guidelines and treatment protocols. Furthermore, to our knowledge no research in humans is available and no similar cases have been published yet in relation to this topic.

In non-pregnant women benzodiazepines are a known treatment option for illicit GHB withdrawal symptoms often in high doses, probably because illicit GHB binds to GABAb receptors, whereas benzodiazepines bind to GABAa receptors. ${ }^{13} 14$ In addition, the GABAb receptor agonist sodium oxybate is regularly used in non-pregnant women in Europe. ${ }^{15}$ Furthermore, the GABAb receptor agonist baclofen seems a logical treatment option, however, little literature is known in regards to successfully treating illicit GHB withdrawal symptoms. ${ }^{16}$

The initial choice to treat the illicit GHB withdrawal symptoms with diazepam was made after consultation between the liaison psychiatrist and the obstetrician. Diazepam was chosen as first treatment option in the absence of any knowledge of available scientific evidence on the short-term and long-term effects of sodium oxybate in relation to the treatment of illicit GHB withdrawal symptoms during pregnancy. This lack of knowledge is an important gap in the current scientific literature. In addition, the relatively high resting heart rate, which was not an actual tachycardia, and the troubled sleeping were initially interpreted as a normal phenomenon of the pregnancy.

However, in retrospect these physiological phenomena could also have been classified as withdrawal symptoms. This exemplifies the difficulty of diagnosing the broad spectrum of illicit GHB withdrawal symptoms in a pregnant woman, since there is partial overlap between mild symptomatology secondary to withdrawal and the normal phenomenology in pregnancy. Furthermore, this case shows the dangers of not adequately treating illicit GHB withdrawal symptoms, since this can rapidly result into a severe withdrawal delirium with extremely dangerous behaviour. In addition, illicit GHB withdrawal is known for waxing and waning of its symptom intensity and is characterised by patients who are sometimes asymptomatic for several days, while thereafter presenting with an acute increase in (severe) withdrawal symptoms. ${ }^{3}$ Subsequently, the choice was made to switch to sodium oxybate, instead of increasing the dosage of diazepam, since the delirium was classified as a severe withdrawal symptom. Due to the severity she was treated the same as a non-pregnant woman would have been treated and therefore sodium oxybate was started in accordance with the practice-based protocol by NISPA, that is, the Nijmegen Institute for Scientist-Practitioners in Addiction, which is applied routinely in the hospital where the patient was presented to. ${ }^{17}$

In this case initially diazepam seemed to be effective to treat the illicit GHB withdrawal symptoms, however, the medication may have been tapered-off too quickly. This emphasises the importance of closely monitoring the effects of diazepam and any concurrent signs of withdrawal in a pregnant woman. In addition, this case illustrates to go slow in regard to the tapering-off of diazepam, whenever there are any possible signs of 
withdrawal. Furthermore, this case seems to show that it is safe to administer sodium oxybate during the third term of the pregnancy in case of an inadequate effect by initial treatment with diazepam and to treat severe illicit GHB withdrawal symptoms, since both mother and daughter were discharged from the hospital in a good condition. In addition, pregnant women presented with illicit GHB withdrawal symptoms are already using this drug with all possible effects on their pregnancy, before any treatment with sodium oxybate is even started. Subsequently, we prefer administering sodium oxybate in a controlled hospital setting. Nevertheless, the question remains whether or not administering sodium oxybate as treatment for a short period of time, compared with a benzodiazepine regime, will have any negative clinical effect in regards to the remaining pregnancy. For example, a laboratory study in chick embryos by GuiraoPiñeyro et al. showed that sodium oxybate significantly altered the transverse and vertical cranial measurements in comparison with vehicle-treated controls, even though no significant differences in body and brain weights were seen. ${ }^{18}$ The authors concluded that their study suggests a possible harmful effect of sodium oxybate on cranial development. ${ }^{18}$ Furthermore, in this case we do not know if there is a relationship between sodium oxybate and the pre-eclampsia the patient was suffering from.

We conclude that more research is needed to effectively and safely recognise and treat illicit GHB withdrawal symptoms in pregnant women in order to provide clinicians the necessary information to adequately deal with this critical professional situation.

\section{Learning points}

No evidence-based and practiced-based guidelines and treatment protocols are available for the treatment of illicit gamma-hydroxybutyric acid (GHB) withdrawal symptoms in pregnant women.

- There is a partial overlap between mild illicit GHB withdrawal symptoms and normal phenomenology in pregnancy.

- Inadequate treatment of illicit GHB withdrawal symptoms can rapidly result into a severe withdrawal delirium; this poses tremendous toxic health effects for the pregnant female and her fetus; this also creates a difficult critical professional situation in the absence of evidence-based and practiced-based guidelines and treatment protocols.

Contributors All authors, JCVM, BAGD and ACMV, were involved in the conception and design of the article. Subsequently JCvM was involved in drafting the article, whereas BAGD and ACMV were involved in critically revising the draft of the article. All authors, JCVM, BAGD and ACMV, have given final approval of the version to be published. In addition, JCVM, BAGD and ACMV have agreed to be accountable for the article and to ensure that all questions regarding the accuracy or integrity of the article are investigated and resolved.

Funding The authors have not declared a specific grant for this research from any funding agency in the public, commercial or not-for-profit sectors.
Competing interests None declared.

Patient consent for publication Obtained.

Provenance and peer review Not commissioned; externally peer reviewed.

Open access This is an open access article distributed in accordance with the Creative Commons Attribution Non Commercial (CC BY-NC 4.0) license, which permits others to distribute, remix, adapt, build upon this work non-commercially, and license their derivative works on different terms, provided the original work is properly cited and the use is non-commercial. See: http://creativecommons.org/ licenses/by-nc/4.0/.

\section{ORCID iD}

JC van Mechelen http://orcid.org/0000-0001-6686-9727

\section{REFERENCES}

1 Carter LP, Pardi D, Gorsline J, et al. Illicit gamma-hydroxybutyrate (GHB) and pharmaceutical sodium oxybate (Xyrem): differences in characteristics and misuse. Drug Alcohol Depend 2009;104:1-10.

2 Maitre M. The gamma-hydroxybutyrate signalling system in brain: organization and functional implications. Prog Neurobiol 1997;51:337-61.

3 van Noorden MS, van Dongen LCAM, Zitman FG, et al. Gamma-Hydroxybutyrate withdrawal syndrome: dangerous but not well-known. Gen Hosp Psychiatry 2009;31:394-6.

4 Degenhardt L, Darke S, Dillon P. Ghb use among Australians: characteristics, use patterns and associated harm. Drug Alcohol Depend 2002;67:89-94.

5 Maxwell JC. Party drugs: properties, prevalence, patterns, and problems. Subst Use Misuse 2005:40:1203-40.

6 Zvosec DL, Smith SW, Porrata T, et al. Case series of $226 \gamma$-hydroxybutyrate-associated deaths: lethal toxicity and trauma. Am J Emerg Med 2011;29:319-32.

7 Galicia M, Nogue S, Miró O. Liquid ecstasy intoxication: clinical features of 505 consecutive emergency department patients. Emerg Med J 2011;28:462-6.

8 Brennan R, Van Hout MC. Gamma-Hydroxybutyrate (GHB): a scoping review of pharmacology, toxicology, motives for use, and user groups. J Psychoactive Drugs 2014:46:243-51.

9 Miró Òscar, Galicia M, Dargan P, et al. Intoxication by gamma hydroxybutyrate and related analogues: clinical characteristics and comparison between pure intoxication and that combined with other substances of abuse. Toxicol Lett 2017;277:84-91.

10 Corkery JM, Loi B, Claridge H, et al. Gamma hydroxybutyrate (GHB), gamma butyrolactone (GBL) and 1,4-butanediol (1,4-BD; BDO): a literature review with a focus on UK fatalities related to non-medical use. Neurosci Biobehav Rev 2015;53:52-78.

11 van Amsterdam JGC, van Laar M, Brunt TM, et al. Risk assessment of gammahydroxybutyric acid (GHB) in the Netherlands. Regul Toxicol Pharmacol 2012;63:55-63.

12 European Monitoring Centre for Drugs and Drug Addiction. European drug report 2018: trends and developments. publications office of the European Union. Luxembourg, 2018.

13 Schep LJ, Knudsen K, Slaughter RJ, et al. The clinical toxicology of gammahydroxybutyrate, gamma-butyrolactone and 1,4-butanediol. Clin Toxicol 2012;50:458-70

14 Kamal RM, van Noorden MS, Wannet W, et al. Pharmacological treatment in $\gamma$-hydroxybutyrate (GHB) and $\gamma$-Butyrolactone (GBL) dependence: detoxification and relapse prevention. CNS Drugs 2017;31:51-64.

15 Dijkstra BAG, Kamal R, van Noorden MS, et al. Detoxification with titration and tapering in gamma-hydroxybutyrate (GHB) dependent patients: the Dutch GHB monitor project. Drug Alcohol Depend 2017:170:164-73.

16 LeTourneau JL, Hagg DS, Smith SM. Baclofen and gamma-hydroxybutyrate withdrawal. Neurocrit Care 2008:8:430-3.

17 Van Noorden M, Kamal R, Hübner B, et al. NISPA treatment-based treatment protocol for acute GHB withdrawal in the hospital. Nijmegen NISPA 2012:1-18.

18 Guirao-Piñeyro M, Pascual-Morenilla MT, Roda-Moreno JA, et al. Effects of gammahydroxybutyrate (liquid ecstasy) on the development of the chick cranium. Eur J. Anat 2007;11:85-8. 
Copyright 2019 BMJ Publishing Group. All rights reserved. For permission to reuse any of this content visit https://www.bmj.com/company/products-services/rights-and-licensing/permissions/

BMJ Case Report Fellows may re-use this article for personal use and teaching without any further permission.

Become a Fellow of BMJ Case Reports today and you can:

- Submit as many cases as you like

- Enjoy fast sympathetic peer review and rapid publication of accepted articles

- Access all the published articles

- Re-use any of the published material for personal use and teaching without further permission

Customer Service

If you have any further queries about your subscription, please contact our customer services team on +44 (0) 2071111105 or via email at support@bmj.com.

Visit casereports.bmj.com for more articles like this and to become a Fellow 